\title{
Endotracheal Colonization at Birth Is Associated with a Pathogen-Dependent Pro- and Antiinflammatory Cytokine Response in Ventilated Preterm Infants: A Prospective Cohort Study
}

\author{
JOZEF DE DOOY, MARGARETA IEVEN, WIM STEVENS, ANNEMIE SCHUERWEGH, AND \\ LUDO MAHIEU \\ Department of Pediatrics, Division of Neonatology [J.D.D., L.M.], Department of Microbiology [M.I.], \\ and Department of Immunology [A.S., W.S.], University of Antwerp, Faculty of Medicine, B-2610 Wilrijk, \\ Belgium
}

\begin{abstract}
The possible association between mediators of inflammation such as cytokines and perinatal colonization of the respiratory tract remains unclear. This prospective cohort study evaluated endotracheal colonization in 141 ventilated preterm infants at birth. The relation with cytokine response in the airways and C-reactive protein (CRP) in umbilical blood was investigated. Of the 141 preterm infants enrolled in this study, $37(26 \%)$ were colonized. In addition to traditional pathogens (61\%), commensal species (26\%) and Mycoplasmataceae (13\%) were isolated. Both the pro-inflammatory cytokines IL- $1 \beta$, IL-6, IL-8, and tumor necrosis factor (TNF)- $\alpha$ as well as the antiinflammatory IL-10 are increased in colonized patients in a dose-dependent manner, with the strongest response in neonates colonized with Gram-negative organisms. There was no antimicrobial IL-12p70 response in colonized infants. Commensal flora is associated with the same inflammatory response as traditional pathogens.
\end{abstract}

\section{ABSTRACT}

Although the umbilical cord blood CRP level was significantly higher in neonates with endotracheal colonization, it was highest in those colonized with Gram-negative organisms but still close to normal limits. Microorganisms in the endotracheal fluid of ventilated preterm infants are associated with a pathogen-specific and dose-dependent cytokine response in the airways and systemic CRP response. (Pediatr Res 56: 547-552, 2004)

CFU, colony forming units

Abbreviations

CRIB, clinical risk index for babies

CRP, C-reactive protein

NICU, neonatal intensive care unit

SNAP, score for neonatal acute physiology

TA, tracheal aspirate

TNF- $\boldsymbol{\alpha}$, tumor necrosis factor alpha
There is increasing evidence supporting an association between fetal exposure to chorioamnionitis, early gestational preterm labor, and respiratory tract colonization $(1,2)$. Moreover, chorioamnionitis has been associated with an adverse perinatal outcome such as cerebral palsy, chronic lung disease, and mortality in preterm infants $(3,4)$. A wide variety of microorganisms are involved in the pathogenesis of chorioamnionitis (e.g. Escherichia coli, Mycoplasmataceae such as Ureaplasma urealyticum, and anaerobic organisms) $(5,6)$. Prenatal diagnosis of chorioamnionitis remains difficult. An early diagnosis can only be made in $6-24 \%$ of these patients by amniotic fluid culture alone (7). Recently, we demonstrated that TA pro-inflammatory cytokines (e.g. IL-8) measured im-

Received May 21, 2003; accepted March 22, 2004.

Correspondence: Jozef De Dooy, M.D., University Hospital Antwerp, Department of Pediatrics, Division of Neonatology, Wilrijkstraat 10, B-2650 Edegem, Belgium; e-mail: jd978722@planetinternet.be

DOI: 10.1203/01.PDR.0000139408.89119.F2 mediately after birth are significantly increased in infants born from mothers with histologic chorioamnionitis (8).

Besides hyaline membrane disease due to a lack of alveolar surfactant, neonatal pneumonia is a well-described cause of respiratory insufficiency (9). Excessive pro-inflammatory stimulation or the lack of antiinflammatory suppression in the lungs of developmentally immature infants may perpetuate the complex inflammatory cytokine response resulting in acute and/or long-term multisystem morbidity $(10,11)$. Cytokines are a group of low-molecular-weight polypeptides involved in cellto-cell communication, including immune function and inflammatory responses after acute and chronic stimuli. The complex interaction between mediators of inflammation such as cytokines and their possible association with perinatal colonization of the lower respiratory tract remains unclear.

Therefore, the objective of this study was to determine the effect of endotracheal colonization at birth on the early postnatal inflammatory response in the lower respiratory tract by 
cytokine analysis in ventilated preterm newborns. This is the first study to specifically assess the association between endotracheal colonization with organisms other than Ureaplasma spp. and the inflammatory response in the lung of ventilated preterm infants.

\section{METHODS}

Study population. A cohort study of 141 preterm infants with a gestational age $<31$ wk requiring ventilation immediately after birth was prospectively studied. The following criteria for ventilation were applied: in the delivery room, infants were deemed surfactant deficient if, in the opinion of the attending clinician, they had clinical signs of respiratory distress (persistent cyanosis after bag ventilation, grunting, severe subcostal indrawing) and/or at the NICU when respiratory acidosis (arterial $\mathrm{P}_{2}>50 \mathrm{~mm} \mathrm{Hg}$ with $\mathrm{pH}<7.25$ on two occasions with an interval of at least $2 \mathrm{~h}$ or a $\mathrm{pH}<7.20$ on one occasion) on blood gas analysis was found. Patients needing mechanical ventilation for nonpulmonary reasons (e.g. congenital malformations such as cardiac defects, abdominal wall defects, and gastrointestinal defects including hernia diaphragmatica) were excluded. All infants were admitted to the NICU of the University Hospital of Antwerp (Edegem, Belgium) between September 1999 and March 2002. Perinatal characteristics of the neonates were registered. These included gender, gestational age, birth weight, Apgar score at $5 \mathrm{~min}$, respiratory distress syndrome according to the radiologic criteria of Edwards (12), occurrence of early onset sepsis, and two scores for assessing the severity of illness [SNAP (13) and CRIB score (14)]. Recorded pregnancy-related maternal data were maternal antibiotic treatment, pregnancy-induced hypertension, histologic chorioamnionitis, preterm premature rupture of membranes, cesarean section, and multiple pregnancy. The research ethics committee gave approval for the study and written parental informed consent was obtained before patients were enrolled.

TA sampling. Although TA sampling is not an optimal way to look at lung processes, it is the only way that it can be done in preterm ventilated neonates. In experimental models of chorioamnionitis, the epithelium of the airways can express cytokines (15). Therefore, we assume that the TA results reflect lung parenchymal responses. TA were performed as soon as possible after birth but always within $2 \mathrm{~h}$ and before administration of surfactant. The TA were performed in a standard manner by the same operator (J.D.D.) with assistance of the nursing staff. A closed tracheal suction system (Trach Care, Ballard Medical Products, Draper, UT) was used, allowing sterile suctioning and avoiding ventilator disconnection. The infant's head was turned to the left and $1 \mathrm{~mL}$ sterile $0.9 \%$ saline was instilled down the endotracheal tube. After five mechanical breaths or $10 \mathrm{~s}$ of high-frequency ventilation, aspiration of tracheal fluid was performed positioning the catheter tip about $1 \mathrm{~cm}$ beyond the end of the endotracheal tube. The infant's head was then turned to the midline and to the right, at each position repeating the aspiration without instilling saline. The whole procedure was then repeated. The median volume returned was $1.7 \mathrm{~mL}$ (range, $1.5-1.9 \mathrm{~mL}$ ).
During the study period, 199 infants below 31 wk of gestation were admitted to the NICU. Of these infants, 158 (79\%) were ventilated at admission. All these infants were enrolled in the study, except for those born during the researcher's (J.D.D.) vacation periods $(17,11 \%)$. Thus, 141 out of $158(89 \%)$ of the ventilated infants below $31 \mathrm{wk}$ gestation were enrolled in this study. In all cases, we were able to collect enough TA sample fluid for further analyses. Furthermore, none of the parents refused enrollment of their infant in the study.

TA cultures. An aliquot of the TA was used for microbiological examination. The sample was sent to the clinical microbiology laboratory for quantitative aerobic and anaerobic bacterial cultures including Ureaplasma spp. according to standard techniques. Results are expressed as CFU per milliliter.

Chorioamnionitis. Histologic chorioamnionitis was diagnosed by light microscopy and registered in a standardized protocol (16). HC was characterized by $>10$ polymorphonuclear granulocytes per high-power field in the subchorion, chorion, or amnion (17).

Neonatal early onset sepsis. At time of admission, a blood culture was taken through the umbilical venous catheter (Bactec system, Becton, Dickinson, Franklin Lakes, NJ). Early-onset sepsis was defined by the detection of a positive blood culture within $72 \mathrm{~h}$ after birth. After the blood culture was drawn and according to the protocol for ventilated preterm neonates in our NICU, all neonates were treated with ampicillin plus netilmicin.

TA cytokine determination. After removing some TA fluid for bacterial cultures, the remaining TA sample was centrifuged for $10 \mathrm{~min}$ at $160 \mathrm{~g}$ at $4^{\circ} \mathrm{C}$ and the supernatant stored at $-80^{\circ} \mathrm{C}$ for subsequent analysis. In TA samples, IL- $1 \beta$, IL-8, IL-6, IL-10, IL-12p70, and TNF- $\alpha$ were measured. Cytokines were determined using a flow cytometric microsphere-based immunoassay, the cytometric bead array (CBA) (BD Biosciences, San Jose, CA) as described by Cook et al. (18). Details on the cytokine determination were described previously (8).

Determination of $\boldsymbol{C R P}$. Results on CRP $(\mathrm{mg} / \mathrm{dL})$ were obtained on umbilical cord blood samples using the VITROS950 analyzer (Ortho-Clinical Diagnostics, Raritan, NJ).

Statistical analysis. Data were collected using the program Filemaker Pro 5.0 (Filemaker, Inc., Santa Clara, CA). Analysis was done with the statistical package Stata 7.0 (Stata Corporation, College Station, TX). Yates corrected $\chi^{2}$ analysis was used for categorical data and Kruskal-Wallis test for nonparametric continuous data. Univariate linear regression analysis was performed to identify a relation between the number of CFU per milliliter of the isolated pathogens and cytokine concentrations in the TA. The relation between TA inflammatory parameters and umbilical cord blood CRP was analyzed with univariate and multiple linear regression analysis. Correlation with $p$ values $<0.05$ was regarded as significant. Results for continuous data are presented as median with $25 \%$ (Q1) and $75 \%$ (Q3) quartiles. Umbilical cord blood CRP levels are expressed as mean $\pm \mathrm{SD}$. 


\section{RESULTS}

Study population. Perinatal and pregnancy-related characteristics were similar in the group with and without endotracheal colonization except for histologic evidence of chorioamnionitis $[26 / 30(87 \%)$ in the group with endotracheal colonization versus $34 / 89(38 \%)$ in the group without endotracheal colonization, $\chi^{2}=21, p<0.0001$ ], preterm premature rupture of membranes [19/37 (51\%) in the group with endotracheal colonization versus $22 / 104(22 \%)$ in the group without endotracheal colonization, $\chi^{2}=12, p<0.001$ ], and pregnancy-induced hypertension $[2 / 37(5 \%)$ in the group with endotracheal colonization versus $33 / 103(32 \%)$ in the group without endotracheal colonization, $\chi^{2}=10, p=0.0013$ ] (Table 1). Of the 141 preterm infants enrolled in this study, $37(26 \%)$ had a positive TA culture. Isolated organisms were Gram-negative organisms (24\%): E. coli (13\%), Enterobacter cloacae (5\%), Haemophilus influenzae (3\%), Klebsiella pneumoniae (3\%); Gram-positive organisms (25\%): Streptococcus agalactiae (11\%), Staphylococcus aureus (8\%), Enterococcus faecalis (3\%), Streptococcus pneumoniae (3\%); Candida spp. 11\%; Mycoplasmataceae (14\%): Mycoplasma spp. (3\%), Ureaplasma spp. (11\%); commensal flora (26\%): viridans streptococci (5\%), Micrococcus spp. (3\%), coagulase-negative staphylococci (15\%), and Capnocytophaga spp. (3\%). There were no infants positive for multiple organisms and no anaerobic organisms were cultured.

Relationship between tracheal colonization and lung cytokine concentrations. Only the median (quartile Q1-Q3) IL$12 \mathrm{p} 70$ concentration measured in the TA was not significantly different between the group with endotracheal colonization and

Table 1. Early perinatal and pregnancy-related characteristics of ventilated preterm infants with and without endotracheal colonization

\begin{tabular}{lcc}
\hline & $\begin{array}{c}\text { TA culture } \\
\text { positive } \\
26 \%(n=37)\end{array}$ & $\begin{array}{c}\text { TA culture } \\
\text { sterile } \\
74 \%(n=104)\end{array}$ \\
\hline Demographic & & \\
Gender: male & $57(21)^{*}$ & $46(48)$ \\
SGA & $5(2)$ & $18(19)$ \\
Gestational age (wk) & $28(26-29) \dagger$ & $28.4(27-29.7)$ \\
Birth weight (g) & $1077(885-1300)$ & $963(807-1233)$ \\
Clinical & & \\
Apgar score at 5 min & $8(7-8)$ & $8(7-8)$ \\
RDS & $76(28)$ & $82(85)$ \\
Early onset sepsis & $0(0)$ & $0(0)$ \\
CRIB score & $5(2-9)$ & $5(2-7)$ \\
SNAP score & $15(10-19)$ & $13(9-17)$ \\
Pregnancy related & & \\
Maternal antibiotic treatment & $65(24)$ & $53(55)$ \\
Histologic chorioamnionitis & $87(26 / 30)$ & $38(34 / 89) \dagger$ \\
PPROM & $51(19)$ & $22(22) \dagger$ \\
Pregnancy-induced hypertension & $5(2)$ & $32(33) \dagger$ \\
Multiple pregnancy & $22(8)$ & $33(34)$ \\
Cesarean section & $50(18)$ & $54(54)$ \\
\hline
\end{tabular}

TA, tracheal aspirate; SGA, small for gestational age; HFV, high-frequency ventilation; IVH, intraventricular hemorrhage according to Papile's classification; RDS, respiratory distress syndrome; PPROM, preterm premature rupture of membranes.

* Percentage (number); $\dagger$ Median $(\mathrm{Q} 1-\mathrm{Q} 3) ;+p<0.05\left(\chi^{2}\right.$ test with Yate's correction). the group without endotracheal colonization $[9(6-16)$ versus $10(6-15) \mathrm{pg} / \mathrm{mL}, p=0.9]$. All the other median (quartile Q1-Q3) cytokine concentrations were significantly higher $(p<$ $0.05)$ in the group with a positive TA culture when compared with the group with a sterile TA culture: IL-1 $\beta, 178(64-426)$ versus $59(45-104) \mathrm{pg} / \mathrm{mL}(p=0.0001)$; IL-6, $3846(111-$ $11,066)$ versus $915(354-2979) \mathrm{pg} / \mathrm{mL}(p=0.0003)$; IL-8, $4071(1475-8092)$ versus $630(294-2388) \mathrm{pg} / \mathrm{mL}(p=$ $0.0001)$; IL-10, 31 (18-73) versus $22(14-37) \mathrm{pg} / \mathrm{mL}(p=$ $0.011)$; TNF- $\alpha, 29$ (12-38) versus 8 (6-16) $\mathrm{pg} / \mathrm{mL}(p=$ 0.0001). Cytokine concentrations in the TA for the different groups of microorganisms isolated in the TA were compared with the group of patients without endotracheal colonization (Table 2). Compared with patients with a sterile TA, the pro-inflammatory cytokines IL-1 $\beta$, IL-6, IL-8, and TNF- $\alpha$ were significantly increased and highest in patients with a Gram-negative pathogen in their TA. The antibacterial cytokine IL-12 was not different in colonized patients when compared with those with a sterile TA culture. The same proinflammatory response, although less pronounced, was found in patients with a Gram-positive pathogen and in those with commensal species in the TA. Remarkably, the antiinflammatory cytokine IL-10 response was only significantly increased in neonates colonized with Gram-negative pathogens. The cytokines IL- $1 \beta$ and IL- 8 are also significantly higher in patients with commensal flora isolated in the TA when compared with patients with a sterile TA culture. The presence of Candida spp. and Mycoplasmataceae did not result in differences in cytokine levels in comparison with patients with a sterile TA culture.

Relationship between endotracheal colonization, lung inflammatory cytokines, and umbilical cord blood CRP response. The umbilical cord blood CRP levels were significantly higher in the group with endotracheal colonization when compared with neonates without endotracheal colonization (mean CRP, $0.7 \pm 1.3$ versus $0.4 \pm 1.7 \mathrm{mg} / \mathrm{dL}, \chi^{2}=6.4, p=$ 0.012). The umbilical blood CRP response was different according to the group of microorganisms involved. Only in neonates colonized with a Gram-negative pathogen umbilical cord blood CRP level was significantly higher than in noncolonized infants (Gram-negative pathogens: mean CRP, $1.1 \pm 1.2$ versus $\left.0.4 \pm 1.7 \mathrm{mg} / \mathrm{dL}, \chi^{2}=8.7, p=0.003\right)$. Colonization by Gram-positive pathogens, commensal flora, Candida spp., or Mycoplasmataceae had no significant effect on the umbilical cord blood CRP level (Gram-positive pathogens: mean CRP, $1.0 \pm 2.1$ versus $0.4 \pm 1.7 \mathrm{mg} / \mathrm{dL}, \chi^{2}=1.7, p=0.19$; commensal flora: mean CRP, $0.7 \pm 1.0$ versus $0.4 \pm 1.7$ $\mathrm{mg} / \mathrm{dL}, \chi^{2}=1.8, p=0.18$; Candida spp.: mean CRP, $0.5 \pm$ 0.9 versus $0.4 \pm 1.7 \mathrm{mg} / \mathrm{dL}, \chi^{2}=0.26, p=0.61$; Mycoplasmataceae: mean CRP, $0.0 \pm 0.0$ versus $0.4 \pm 1.7 \mathrm{mg} / \mathrm{dL}, \chi^{2}=$ $1.03, p=0.31)$. Univariate linear regression analysis revealed an association between umbilical cord blood CRP and the cytokines IL-1 $\beta(r=0.29-1.07, p=0.001)$, IL-6 $(r=$ $0.15-0.87, p=0.006)$, IL-8 $(r=0.30-0.99, p=0.001)$, IL-10 $(r=0.18-1.17, p=0.007)$, and TNF- $\alpha(r=0.00001-$ $0.0005, p=0.036)$. No association was found between umbilical cord blood CRP and IL-12p70 ( $r=-0.15-1.08, p=$ $0.14)$. After multivariate regression analysis, only IL- 8 re- 
Table 2. Tracheal aspirate cytokine concentrations in ventilated preterm infants: comparison of noncolonized infants vs colonized infants stratified by group of micro-organisms involved

\begin{tabular}{|c|c|c|c|c|c|c|}
\hline \multirow[b]{2}{*}{ Cytokine } & \multicolumn{6}{|c|}{ Group of micro-organisms in colonized infants } \\
\hline & $\begin{array}{c}\text { Sterile } \\
(n=104)\end{array}$ & $\begin{array}{l}\text { Candida spp. } \\
\quad(n=4)\end{array}$ & $\begin{array}{c}\text { Gram-negative } \\
\text { pathogens } \\
(n=9)\end{array}$ & $\begin{array}{c}\text { Gram-positive } \\
\text { pathogens } \\
(n=9)\end{array}$ & $\begin{array}{l}\text { Commensal } \\
\text { flora }(n=10)\end{array}$ & $\begin{array}{c}\text { Mycoplasmataceae } \\
(n=5)\end{array}$ \\
\hline $\mathrm{IL}-1 \beta(\mathrm{pg} / \mathrm{mL})$ & $59(45-104)$ & $98(55-176)$ & $425(207-2520)^{*}$ & $146(97-309) *$ & $145(55-380)^{*}$ & $139(61-178)$ \\
\hline IL-6 (pg/mL) & $915(354-2979)$ & $1747(875-6724)$ & $11896(1568-43260)^{*}$ & $4187(2083-4933)^{*}$ & $3621(960-7287)$ & $728(497-4520)$ \\
\hline IL-8 (pg/mL) & $630(294-2388)$ & $2292(1272-3589)$ & $15735(3548-75656)^{*}$ & $4967(3537-7930) *$ & $2626(1050-4607)^{*}$ & $3829(580-4995)$ \\
\hline $\mathrm{TNF}-\alpha(\mathrm{pg} / \mathrm{mL})$ & $8(6-16)$ & $23(9-37)$ & $164(20-1510)^{*}$ & $35(23-55)^{*}$ & $11(9-29)$ & $12(11-29)$ \\
\hline IL-10 (pg/mL) & $22(14-37)$ & $27(14-64)$ & $224(29-468) *$ & $31(24-55)$ & $35(16-41)$ & $24(20-33)$ \\
\hline IL-12p70 (pg/mL) & $10(6-15)$ & $8(6-13)$ & $12(5-32)$ & $12(8-14)$ & $9(7-16)$ & $6(0-7)$ \\
\hline
\end{tabular}

$* p<0.05$ when compared with patients with a sterile TA culture (Kruskal-Wallis test).

mained correlated with the umbilical cord blood CRP $(r=$ $0.30-0.99, p=0.001$ ).

Quantitative bacterial cultures. The number of CFU per milliliter TA in decreasing order in the different groups of micro-organisms were [median (Q1-Q3)]: Candida spp., 800 (250-1000) CFU/mL; Gram-positive pathogens, 750 (2351500) CFU/mL; Gram-negative pathogens, 575 (250-1000) $\mathrm{CFU} / \mathrm{mL}$; commensal flora, 310 (70-1000) CFU/mL; Mycoplasmataceae, $10(10-90,900) \mathrm{CFU} / \mathrm{mL}$. A positive relationship was found between the number of CFU per milliliter and the cytokines IL- $1 \beta(y=0.092 x+1.804, r=0.38, p=$ $0.0001)$, IL-6 $(y=0.080+3.003, r=0.31, p=0.0002)$, IL-8 $(y=0.115+2.923, r=0.42, p=0.0001), \mathrm{IL}-10(y=0.059 x$ $+1.336, r=0.24, p=0.004)$, and TNF- $\alpha(y=0.096 x+$ 1.022, $r=0.42, p=0.0001)$. There was no correlation between the number of CFU per milliliter and IL-12p70 ( $y=$ $-0.002+0.937, r=-0.02, p=0.79)$.

\section{DISCUSSION}

The main finding of this study is that endotracheal colonization at birth is associated with a significant pro-inflammatory response in the lung in ventilated preterm infants accompanied by a systemic inflammatory CRP response. In addition, this inflammatory response is also observed in neonates colonized with nontraditional pathogens such as coagulase-negative staphylococci. Third, these neonates reveal a strong antiinflammatory cytokine (IL-10) response in the lung, especially in Gram-negative pneumonia. Finally, they do not show any anti-microbial cytokine (IL-12p70) response.

Besides the traditional neonatal pathogens isolated from the lungs of the neonates, we find a high rate of commensal species $(26 \%)$ and just few Mycoplasmataceae (14\%). It is remarkable that we did not find anaerobic microorganisms, which are also important in the pathogenesis of ascending infection in pregnant women (19). Maybe the anaerobic conditions of the aspirated specimen were not adequate to allow detection of anaerobic organisms in the laboratory.

We cannot exclude that the origin of the measured TA cytokines is from mother or fetus inasmuch as a fetus under stressful condition often aspirate amniotic fluid in utero. However, we have reason to believe that the origin of the measured TA cytokines is, at least to a large extent, of fetal origin as an antimicrobial reaction. In colonized neonates, umbilical cord blood CRP levels are significantly higher when compared with noncolonized patients. We further find a moderate but statistically significant correlation between the pathogen doses (CFU/ $\mathrm{mL}$ ) and the cytokines IL- $1 \beta$, IL-6, IL-8, IL-10, and TNF- $\alpha$ in the TA. Although this supports the belief that the airway colonization is responsible for the inflammatory response, the rather low correlation coefficient we find indicates that the majority of the variability in the cytokine response is not explained by variation in CFU per milliliter of the pathogens isolated in the TA. An extra argument for a fetal response to microbial invasion is the significant albeit moderate correlation between TA cytokine concentration and CRP level in umbilical blood. However, we must note that the higher CRP value in colonized neonates is close to the normal clinical limits (1 $\mathrm{mg} / \mathrm{dL}$ ) and that the sensitivity of CRP taken close after birth is low in patients with invasive bacterial disease (20). Presence of positive blood cultures could have supported our arguments for fetal infection but we did not find positive blood cultures in any of the infants studied. However, it is known that the sensitivity of blood cultures in newborn infants is low, especially in those with pneumonia (21). In a previous study we found a strong association between lung inflammation and umbilical vasculitis in patients born from mothers with histologic chorioamnionitis (8), indicating that the measured cytokines most likely are of fetal origin. We have taken these arguments into account, and we suggest that chorioamnionitis is the leading cause for the endotracheal colonization we observed and that the subsequent inflammatory reaction is for a major part of fetal origin.

Our data show that the inflammatory cytokine response is highest in neonates colonized with a Gram-negative pathogen. This can be explained by the lipopolysaccharide (LPS) and/or flagellin-induced production of pro-inflammatory cytokines in Gram-negative bacteria $(22,23)$. Furthermore, the antiinflammatory cytokine IL-10 was only significantly increased in neonates colonized with a Gram-negative pathogen. It is known from animal models that IL-10 attenuates the proinflammatory cytokine response within the lungs, hampers effective clearance of the infection, and shortens survival (24). These mechanisms can explain why we observe the highest inflammatory cytokine response in neonates colonized with Gram-negative organisms. In accordance with this pronounced inflammatory cytokine response, umbilical cord blood CRP is also significantly increased in these neonates. 
In the present study, we find a significant pro-inflammatory response in patients colonized with Gram-positive pathogens (e.g. S. agalactiae), although less pronounced than in Gramnegative colonized patients. The mechanisms by which Grampositive bacteria cause the release of pro-inflammatory cytokines such as IL- $1 \beta$, IL-6, IL-8, and TNF- $\alpha$ from human neonatal cells are not very clear. Gram-positive cell wall components such as lipoteichoic acids are known to be strong activators of the macrophage/monocyte system, even in neonates $(25,26)$. We also find an increased CRP value in umbilical cord blood of neonates colonized with Gram-positive organisms, but it does not reach statistical significance, maybe due to the limited number of cases (type II error).

Our data show that lower respiratory tract colonization with commensal flora (e.g. coagulase-negative staphylococci) is also associated with a local inflammatory cytokine response. The association of commensal flora with neonatal pneumonia has not yet been elucidated, but it is known that these organisms may behave like classic nosocomial pathogens, causing ventilator associated pneumonia in critically ill and immunocompromised adults $(27,28)$. Moreover, these organisms have been found in maternal genital tract infection complicated with chorioamnionitis (29). Therefore, we suggest that these organisms must be considered as significant pathogens rather than colonizers in the immunocompromised preterm neonate (30). Furthermore, there is also an increased CRP value in the umbilical cord blood of neonates colonized with commensal organisms. Again, probably due to the limited number of cases, statistical significance was not reached (type II error).

Ureaplasma spp. was not frequently isolated in the lungs of the neonates. However, in other studies, colonization rates were clearly higher (up to 45\%) (19). This difference can reflect a lower infection rate among our study population or can be due to the low sensitivity of the culture method used. Indeed, we did not use the more sensitive polymerase chain reaction technique (31). Moreover, according to hospital policy, the majority of the mothers with preterm premature rupture of membranes are treated with azithromycin, an agent active against Ureaplasma spp. We do not find a significant increase of cytokines in the lungs nor in umbilical cord blood CRP of neonates colonized with Ureaplasma spp. and Candida spp., suggesting that these organisms rather colonize the airways instead of causing lung infection. Indeed, it is still controversial whether colonization with Ureaplasma spp. is associated with an increased inflammatory response in the lungs of preterm infants and with adverse neonatal outcome such as chronic lung disease (32-34). Paradoxically, a recent study in mice showed an important role of alveolar macrophage-mediated cytokine response in Candida-induced acute lung injury (35).

A remarkable but consistent finding among all the groups of pathogens isolated was that the antimicrobial cytokine IL12 p70 level was not different when compared with noncolonized neonates. Preterm infants lack IL-12p70 response for several reasons: deficiencies in proteins (e.g. CD40) that participate in macrophage activation (36), an insufficient T-cell activity as a consequence of intrauterine stress involution of the thymus secondary to chorioamnionitis (37), and deficiencies in the number and/or function of dendritic cells in the neonatal period (38).

We could not isolate any anaerobic bacteria in the TA samples. However, it is known that these microorganisms are considered to be important in the pathogenesis of chorioamnionitis (39). In a previous study, we found a significantly increased inflammatory lung cytokine response in patients with chorioamnionitis. Moreover, chorioamnionitis was found in $15 \%$ of patients with a sterile TA culture (8). We cannot exclude that the cytokine response measured in patients with a sterile TA is caused by the presence of anaerobic organisms. Therefore, the real difference of the inflammatory response between TA culture positive and negative patients might even be more prominent.

In conclusion, we demonstrated in this study that preterm colonized neonates develop a pathogen-dependent proinflammatory cytokine response in their lower respiratory tract. The meaning of this immunologic reaction on neonatal outcome, including the more pronounced production of the antiinflammatory cytokine IL-10 in patients colonized with Gramnegative organisms and the lack of the antimicrobial cytokine IL-12p70 response in all colonized infants needs to be clarified. Although the pathogen-induced lung inflammatory reaction is associated with a systemic CRP response, the sensitivity of CRP of umbilical cord blood is too low to discriminate lung infection from airway colonization. A study of the association between airway colonization and the inflammatory response in the lower respiratory tract on the neonatal outcome will not only allow a better understanding of the pathogenesis of diseases but will also be helpful in developing new therapeutic approaches in ventilated preterm infants with respiratory distress syndrome.

Acknowledgments. The authors thank Dr. Cecile Colpaert (Pathology, University Hospital Antwerp, Belgium) for her help in the standardized diagnosis of histologic chorioamnionitis. We also thank Mr. Chris Bridts (Laboratory of Clinical Immunology, University Hospital Antwerp, Belgium) for his assistance on cytokine determination in the tracheal aspirates.

\section{REFERENCES}

1. Goldenberg RL, Hauth JC, Andrews WW 2000 Intrauterine infection and preterm delivery. N Engl J Med 342:1500-1507

2. Hannaford K, Todd DA, Jeffery H, John E, Blyth K, Gilbert GL 1999 Role of Ureaplasma urealyticum in lung disease of prematurity. Arch Dis Child Fetal Neonatal Ed 81:F162-F167

3. Leviton A, Paneth N, Reuss ML, Susser M, Allred EN, Dammann O, Kuban K, Van Marter LJ, Pagano M, Hegyi T, Hiatt M, Sanocka U, Shahrivar F, Abiri M, Disalvo D, Doubilet P, Kairam R, Kazam E, Kirpekar M, Rosenfeld D, Schonfeld S, Share J, Collins M, Genest D, Shen-Schwarz S, et al. 1999 Maternal infection, fetal inflammatory response, and brain damage in very low birth weight infants. Pediatr Res 46:566-575

4. Elimian A, Verma U, Beneck D, Cipriano R, Visintainer P, Tejani N 2000 Histologic chorioamnionitis, antenatal steroids, and perinatal outcomes. Obstet Gynecol 96:333336

5. Gibbs RS, Blanco JD, St Clair PJ, Castaneda YS 1982 Quantitative bacteriology of amniotic fluid from women with clinical intraamniotic infection at term. J Infect Dis 145:1-8

6. Shurin PA, Alpert S, Bernard Rosner BA, Driscoll SG, Lee YH 1975 Chorioamnionitis and colonization of the newborn infant with genital mycoplasmas. N Engl J Med 293:5-8

7. Romero R, Yoon BH, Mazor M, Gomez R, Diamond MP, Kenney JS, Ramirez M, Fidel PL, Sorokin Y, Cotton D, Sehgal P 1993 The diagnostic and prognostic value of amniotic fluid white blood cell count, glucose, interleukin-6, and gram stain in 
patients with preterm labor and intact membranes. Am J Obstet Gynecol 169:805816

8. De Dooy J, Colpaert C, Schuerwegh A, Bridts C, Van Der Planken M, Ieven M, De Clerck L, Stevens W, Mahieu L 2003 Relationship between histologic chorioamnionitis and early inflammatory variables in blood, tracheal aspirates, and endotracheal colonization in preterm infants. Pediatr Res 54:113-119

9. Webber S, Wilkinson AR, Lindsell D, Hope PL, Dobson SR, Isaacs D 1990 Neonatal pneumonia. Arch Dis Child 65:207-211

10. Groneck P, Speer CP 1995 Inflammatory mediators and bronchopulmonary dysplasia. Arch Dis Child Fetal Neonatal Ed 73:F1-F3

11. Ozdemir A, Brown MA, Morgan WJ 1997 Markers and mediators of inflammation in neonatal lung disease. Pediatr Pulmonol 23:292-306

12. Edwards DK, Hilton SV, Merritt TA, Hallman M, Mannino F, Boynton BR 1985 Respiratory distress syndrome treated with human surfactant: radiographic findings. Radiology 157:329-334

13. Richardson DK, Gray JE, McCormick MC, Workman K, Goldmann DA 1993 Score for Neonatal Acute Physiology: a physiologic severity index for neonatal intensive care. Pediatrics 91:617-623

14. The International Neonatal Network 1993 The CRIB (clinical risk index for babies) score: a tool for assessing initial neonatal risk and comparing performance of neonatal intensive care units. Lancet 342:193-198

15. Munshi UK, Niu JO, Siddiq MM, Parton LA 1997 Elevation of interleukin-8 and interleukin-6 precedes the influx of neutrophils in tracheal aspirates from preterm infants who develop bronchopulmonary dysplasia. Pediatr Pulmonol 24:331-336

16. DiSalvo D 1998 The correlation between placental pathology and intraventricula hemorrhage in the preterm infant. The Developmental Epidemiology Network Investigators. Pediatr Res 43:15-19

17. Benirschke K, Kaufmann P 2000 Pathology of the Human Placenta. Springer-Verlag, New York, pp 591-684

18. Cook EB, Stahl JL, Lowe L, Chen R, Morgan E, Wilson J, Varro R, Chan A, Graziano FM, Barney NP 2001 Simultaneous measurement of six cytokines in a single sample of human tears using microparticle-based flow cytometry: allergics vs. non-allergics. J Immunol Methods 254:109-118

19. Boskey ER, Telsch KM, Whaley KJ, Moench TR, Cone RA 1999 Acid production by vaginal flora in vitro is consistent with the rate and extent of vaginal acidification. Infect Immun 67:5170-5175

20. Philip AG 1984 White blood cells and acute phase reactants in neonatal sepsis. Pediatrics 39:371-378

21. Gerdes JS 1991 Clinicopathologic approach to the diagnosis of neonatal sepsis. Clin Perinatol 18:361-381

22. Heumann D, Roger T 2002 Initial responses to endotoxins and Gram-negative bacteria. Clin Chim Acta 323:59-72

23. Liaudet L, Murthy KG, Mabley JG, Pacher P, Soriano FG, Salzman AL, Szabo C 2002 Comparison of inflammation, organ damage, and oxidant stress induced by
Salmonella enterica serovar Muenchen flagellin and serovar Enteritidis lipopolysaccharide. Infect Immun 70:192-198

24. van der Poll T, Marchant A, Keogh CV, Goldman M, Lowry SF 1996 Interleukin-10 impairs host defense in murine pneumococcal pneumonia. J Infect Dis 174:994-1000

25. Keller R, Fischer W, Keist R, Bassetti S 1992 Macrophage response to bacteria: induction of marked secretory and cellular activities by lipoteichoic acids. Infect Immun 60:3664-3672

26. Berner R, Welter P, Brandis M 2002 Cytokine expression of cord and adult blood mononuclear cells in response to Streptococcus agalactiae. Pediatr Res 51:304-309

27. Lambotte O, Timsit JF, Garrouste-Orgeas M, Misset B, Benali A, Carlet J 2002 The significance of distal bronchial samples with commensals in ventilator-associated pneumonia: colonizer or pathogen? Chest 122:1389-1399

28. Reynolds HY 1999 Defense mechanisms against infections. Curr Opin Pulm Med 5:136-142

29. Rosenstein IJ, Morgan DJ, Sheehan M, Lamont RF, Taylor-Robinson D 1996 Bacterial vaginosis in pregnancy: distribution of bacterial species in different gramstain categories of the vaginal flora. J Med Microbiol 45:120-126

30. Schibler KR, Liechty KW, White WL, Rothstein G, Christensen RD 1992 Defective production of interleukin- 6 by monocytes: a possible mechanism underlying several host defence deficiencies of neonates. Pediatr Res 31:18-21

31. Da Silva O, Gregson D, Hammerberg O 1997 Role of Ureaplasma urealyticum and Chlamydia trachomatis in development of bronchopulmonary dysplasia in very low birth weight infants. Pediatr Infect Dis J 16:364-369

32. Patterson AM, Taciak V, Lovchik J, Fox RE, Campbell AB, Viscardi RM 1998 Ureaplasma urealyticum respiratory tract colonization is associated with an increase in interleukin 1-beta and tumor necrosis factor alpha relative to interleukin 6 in tracheal aspirates of preterm infants. Pediatr Infect Dis J 17:321-328

33. Lyon AJ, McColm J, Middlemist L, Ferousson S, McIntosh N, Ross PW 1998 Randomised trial of erythromycin on the development of chronic lung disease in preterm infants. Arch Dis Child Fetal Neonatal Ed 78:F10-F14

34. van Waarde WM, Brus F, Okken A, Kimpen JL 1997 Ureaplasma urealyticum colonization, prematurity and bronchopulmonary dysplasia. Eur Respir J 10:886-890

35. Kubota Y, Iwasaki Y, Harada H, Yokomura I, Ueda M, Hashimoto S, Nakagawa M 2001 Role of alveolar macrophages in Candida-induced acute lung injury. Clin Diagn Lab Immunol 8:1258-1262

36. Grewal IS, Flavell RA 1998 CD40 and CD154 in cell-mediated immunity. Annu Rev Immunol 16:111-135

37. De Felice C, Latini G, Del Vecchio A, Toti P, Bagnoli F, Petraglia F 2002 Small thymus at birth: a predictive radiographic sign of bronchopulmonary dysplasia. Pediatrics 110:386-388

38. Upham JW, Lee PT, Holt BJ, Heaton T, Prescott SL, Sharp MJ, Sly PD, Holt PG 2002 Development of interleukin-12-producing capacity throughout childhood. Infect Immun 70:6583-6588

39. Eschenbach DA 1993 Bacterial vaginosis and anaerobes in obstetric-gynecologic infection. Clin Infect Dis 16:S282-S287 